\title{
A Nearest Level PWM Method for the MMC in DC Distribution Grids
}

Yi Wang ${ }^{1}$, Member, IEEE, Ruoyu Ding ${ }^{1}, \mathrm{Can} \mathrm{Hu}^{1}$, Lie $\mathrm{Xu}^{2}$, Senior Member, IEEE, Chao Fu ${ }^{1}$, Erlin Yang ${ }^{1}$

1. State Key Laboratory of Alternate Electrical Power System with Renewable Energy Sources, North China Electric Power University, Baoding 071003, China

2. Dept. of Electronic \& Electrical Engineering, University of Strathclyde, Glasgow, G1 1XW, UK

Abstract-For modular multilevel converters (MMCs) applied to medium-voltage DC distribution grids, using the traditional Nearest Level Modulation (NLM) as in HVDC systems can lead to severe current distortion due to significantly reduced module number. This paper proposes a hybrid modulation method combining NLM and Pulse Width Modulation (PWM) where only one module per arm operates under PWM mode. The proposed Nearest Level PWM (NL-PWM) method not only significantly reduces the current distortion, but also avoids the complicated voltage balancing control in each module. The harmonic characteristics of NL-PWM are derived using double Fourier transform, which provides theoretical basis for selecting module number and switching frequency for medium-voltage application in accordance with grid harmonic requirements. Finally, the harmonic characteristics and feasibility of the proposed modulation method are validated by simulation and experimental studies on a MMC with 6 modules per arm. The simulated and experimental results reveal that NL-PWM has better voltage and current harmonic characteristics over NLM and CPS-PWM, thereby suiting the application of MMC with few models.

Index Terms-MMC, nearest level modulation, PWM, harmonic analysis, DC grid 
This paper is a post-print of a paper submitted to and accepted for publication in IEEE Transaction on Power Electronics and is subject to Institution of Electrical and Electronic Engineering Copyright. The copy of record is available at IEEE Xplore Digital Library.

\section{INTRODUCTION}

In recent years, modular multilevel converter (MMC) has experienced fast development in a broad application prospects, e.g. DC transmission, solid state transformers, and renewable energy integration, due to its advantages of easy power/voltage expansion and low harmonic contents [1]-[2]. However, for application of medium-voltage (MV) DC distribution grids, there will be big increases in the staircase approximation error and voltage step due to the small module number. As a result, significant low-order voltage harmonics and current distortion could appear which deteriorates the power quality and make it difficult to meet the requirements of distribution grids.

The modulation method has important influence on MMC performances, such as the switching loss, capacitor voltage balancing, and voltage harmonic contents [3]-[4]. Currently, there are two kinds of popular modulation methods for MMC, i.e., Nearest Level Modulation (NLM) and Carrier Phase-Shift PWM (CPS-PWM). In [5]-[9], the principle of NLM is introduced and its implementation is given in detail. The principle of CPS-PWM is presented in [10]-[16], and the voltage balancing and current averaging control are proposed. A novel strategy based on the CPS-PWM is proposed in [17] to implement fault-tolerant control of MMC by replacing failed sub-modules (SMs) with redundant SMs simultaneously. In [18] a flexible capacitor voltage control strategy based on CPS-PWM modulation for MMC for motor drives is proposed. However, the voltage balancing control is complicated under CPS-PWM and the switching loss increases as well.

For MMC used in HVDC transmission with hundreds of series modules, an ideal sinusoidal current can be obtained under the staircase voltage modulated by NLM. However, for MVDC distribution networks below 10kV, only a few SMs are connected in series in each arm, and consequently, the small number of voltage levels result in significant low-order voltage harmonics. Although the voltage harmonics and current distortion can be reduced by applying CPS-PWM, the voltage balancing and current averaging control becomes more complicated since each SM is independently modulated. Its voltage balancing control has to be achieved by regulating the reference wave of each SM, and consequently, the modulation using different reference waves with phase-shifted carriers may cause additional harmonics.

In order to expand the application of MMCs to MVDC distribution networks, this paper proposes a new modulation method to reduce current distortion. Based on the analysis of NLM and CPS-PWM methods, the modulation principle and implementation of the newly proposed PWM method are elaborated. To describe the harmonic characteristics of the proposed PWM method, the analytical expressions of the output voltage harmonics are derived using double Fourier transform. Finally, MMC performance and harmonics under different modulations are compared by simulations, and the feasibility of the proposed modulation method is verified by prototype experiments. 
This paper is a post-print of a paper submitted to and accepted for publication in IEEE Transaction on Power Electronics and is subject to Institution of Electrical and Electronic Engineering Copyright. The copy of record is available at IEEE Xplore Digital Library.

\section{MMC TOPOLOGY AND Modulation Methods}

\section{A. MMC topology}

A three-phase MMC topology is shown in Fig. 1. It has six arms and each arm consists of $N$ sub-modules (SMs) and an inductor $L_{\mathrm{s}}$. This paper only concerns on the half-bridge SM (HBSM) based MMC. The normal switching states of the HBSM are listed in Table I.

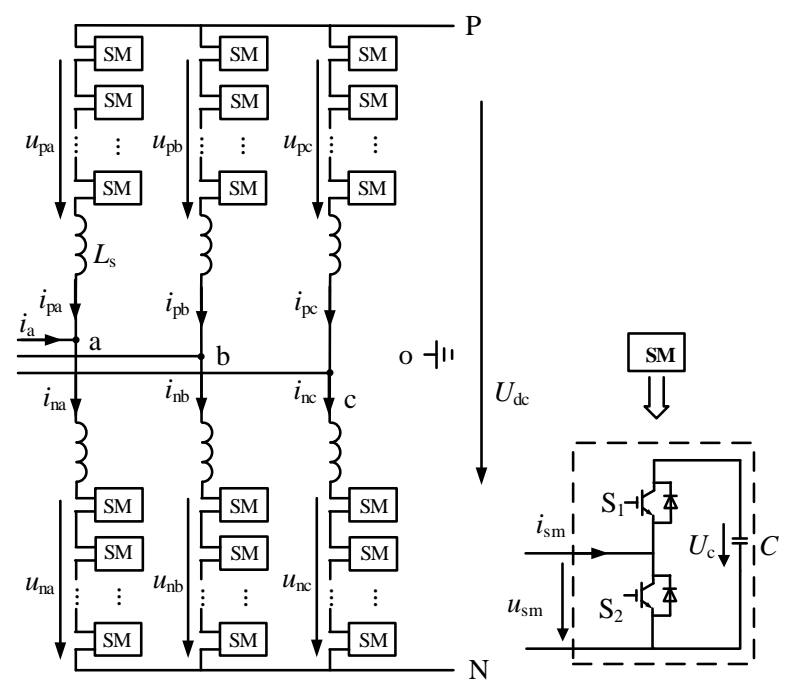

Fig. 1 MMC topology

TABLE I SWITCHING STATES OF SM

\begin{tabular}{cccccc}
\multicolumn{7}{c}{ SWITCHING STATES OF SM } \\
\hline \hline Mode & $\mathrm{S}_{1}$ & $\mathrm{~S}_{2}$ & $i_{\mathrm{sm}}$ & $u_{\mathrm{sm}}$ & State \\
\hline 1 & 1 & 0 & $>0$ & $U_{\mathrm{c}}$ & charging \\
2 & 1 & 0 & $<0$ & $U_{\mathrm{c}}$ & discharging \\
3 & 0 & 1 & $>0$ & 0 & bypass \\
4 & 0 & 1 & $<0$ & 0 & bypass \\
\hline \hline
\end{tabular}

Taking phase $a$ as an example, according to KVL, the voltages of the upper and lower arms of the MMC are expressed as

$$
\begin{aligned}
& u_{\mathrm{pa}}+L_{\mathrm{s}} \frac{\mathrm{d} i_{\mathrm{pa}}}{\mathrm{d} t}=\frac{1}{2} U_{\mathrm{dc}}-u_{\mathrm{ao}} \\
& u_{\mathrm{na}}+L_{\mathrm{s}} \frac{\mathrm{d} i_{\mathrm{na}}}{\mathrm{d} t}=\frac{1}{2} U_{\mathrm{dc}}+u_{\mathrm{ao}}
\end{aligned}
$$

where $u_{\mathrm{pa}}$ and $u_{\mathrm{na}}$ are the total voltages of the cascaded SMs in the upper and lower arms respectively. $i_{\mathrm{pa}}$ and $i_{\mathrm{na}}$ are the currents of the upper and lower arms respectively. $L_{\mathrm{s}}$ is the arm inductor and $u_{\mathrm{io}}$ is the phase to ground voltage which is referred as phase voltage in this paper.

According to (1) and (2), and neglecting the arm inductance voltage, the phase voltage and DC voltage can be expressed as

$$
\begin{gathered}
u_{\mathrm{ao}}=\frac{1}{2}\left(u_{\mathrm{na}}-u_{\mathrm{pa}}\right) \\
U_{\mathrm{dc}}=u_{\mathrm{pa}}+u_{\mathrm{na}} .
\end{gathered}
$$

\section{B. NLM of $M M C$}

The staircase wave is utilized to approximate the desired sine wave by NLM, and the modulation principle is illustrated in Fig. 
This paper is a post-print of a paper submitted to and accepted for publication in IEEE Transaction on Power Electronics and is subject to Institution of Electrical and Electronic Engineering Copyright. The copy of record is available at IEEE Xplore Digital Library.

2. Compared with the high frequency PWM method, NLM has the advantages of less switching loss and easier voltage balancing.

If the number of SMs is large enough, the output voltage and current of the MMC by NLM have good harmonic characteristics.

Therefore, the NLM shows great superiority for HVDC systems above $100 \mathrm{kV}$. As shown in Fig. 2, the phase voltage and arm voltages by NLM are

$$
\begin{gathered}
u_{\mathrm{ao}}=\operatorname{round}\left(\frac{u_{\mathrm{ao}}^{*}}{U_{\mathrm{c}}}\right) \cdot U_{\mathrm{c}} \\
u_{\mathrm{pa}}=N_{\mathrm{pa}} U_{\mathrm{c}} \\
u_{\mathrm{na}}=N_{\mathrm{na}} U_{\mathrm{c}}
\end{gathered}
$$

where $\operatorname{round}(x)$ is the function of rounding the nearest integer. $u_{\mathrm{ao}}{ }^{*}$ is the reference voltage of phase a, and $U_{\mathrm{c}}$ is the capacitor voltage of the SM. $N_{\mathrm{pa}}$ and $N_{\mathrm{na}}$ are the numbers of inserted SMs in the upper and lower arms of phase a, respectively.

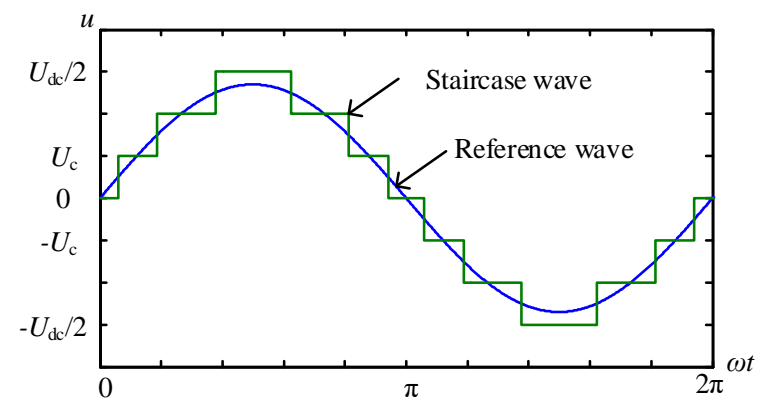

Fig. 2 Principle of NLM strategy

According to (3)-(6), the numbers of the inserted SMs in the upper arm and lower arms can be calculated as

$$
\left\{\begin{array}{l}
N_{\mathrm{pa}}=\frac{N}{2}-\operatorname{round}\left(\frac{u_{\mathrm{ao}}^{*}}{U_{c}}\right) \\
N_{\mathrm{na}}=\frac{N}{2}+\operatorname{round}\left(\frac{u_{\mathrm{ao}}^{*}}{U_{c}}\right)
\end{array}\right.
$$

where $N$ is the number of SMs per arm.

The selection of SMs for inserting also depends on the arm current direction and SM capacitor voltage. While $i_{\mathrm{sm}}>0$, i.e., the capacitors are charging, the lower voltage SMs in the arm are selected first, whereas for $i_{\mathrm{sm}}<0$, the higher voltage SMs are selected first. It can be known that, for the staircase approximation by NLM with only a few voltage levels, the low-order voltage harmonics and current distortion of the MMC will increase significantly.

\section{CPS-PWM of $M M C$}

For CPS-PWM, there are no low-order harmonics in the produced multilevel wave if the switching frequency is sufficiently high. Therefore, the multi-carrier PWM is more suitable for the MV MMC with fewer SMs. The principle of CPS-PWM (N=6) is shown in Fig. 3. The modulation of CPS-PWM is to compare the arm voltage reference with $N$ carriers which have the phase shift of $\pi / N$ in order. Each of the SMs in one arm corresponds to a carrier wave $u_{\mathrm{c} n}$ and all the 6 SMs share the same reference wave $u_{\mathrm{r}}$. If the reference wave is greater than the carrier, the SM corresponding to this carrier is inserted, otherwise, the SM is bypassed. 
This paper is a post-print of a paper submitted to and accepted for publication in IEEE Transaction on Power Electronics and is subject to Institution of Electrical and Electronic Engineering Copyright. The copy of record is available at IEEE Xplore Digital Library.

According to (3), if the voltage reference of phase a is $u_{\mathrm{ao}}^{*}$, the reference waves of the upper and lower arms are $u_{\mathrm{pa}}^{*}=-u_{\mathrm{ao}}^{*}$ and $u_{\mathrm{na}}^{*}=u_{\mathrm{ao}}^{*}$, respectively. Then each of SMs in upper/lower arm modulates independently according to the assigned voltage reference and carrier, and the module rotating for voltage balancing is no longer applicable. Thus, aside from the arm voltage reference, the SM's voltage reference also includes the quantities from voltage balancing control and circulating current averaging control, which is shown in Fig. 4. Unlike the ideal CPS-PWM method shown in Fig. 3, the reference waves of each SM are different in actual modulation, which results in additional harmonics of the MMC.
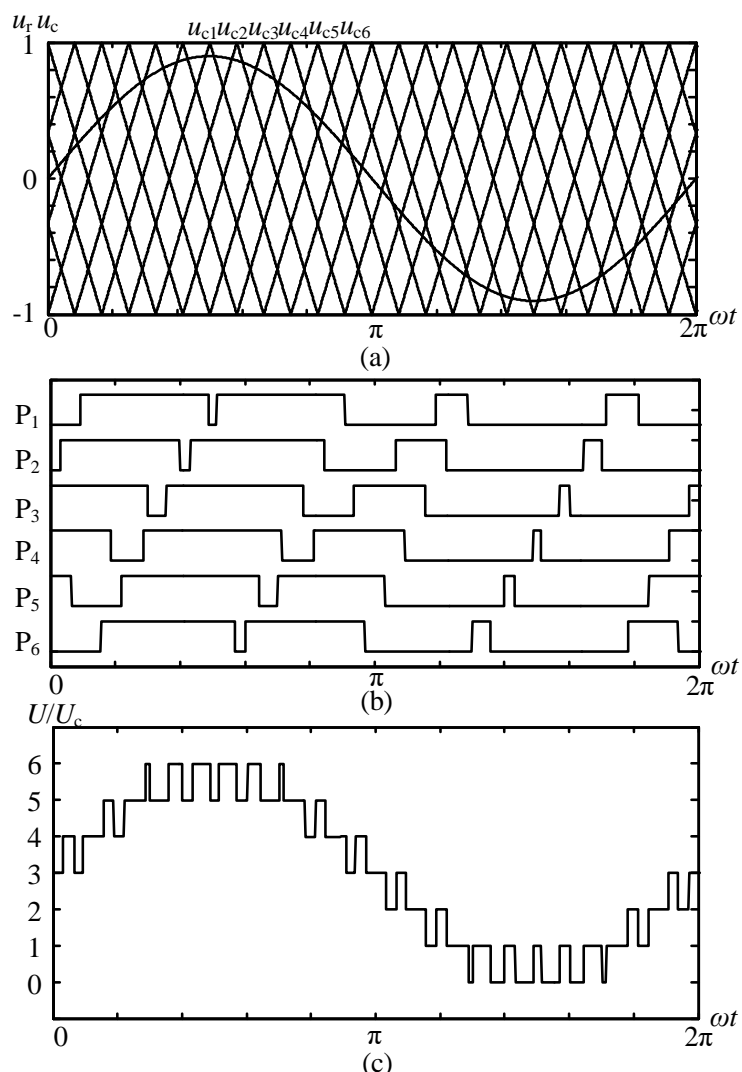

Fig. 3 Principle of CPS-PWM (a) Reference wave and carrier waves (b) Switching signals of SMs in one arm (c) Lower arm voltage

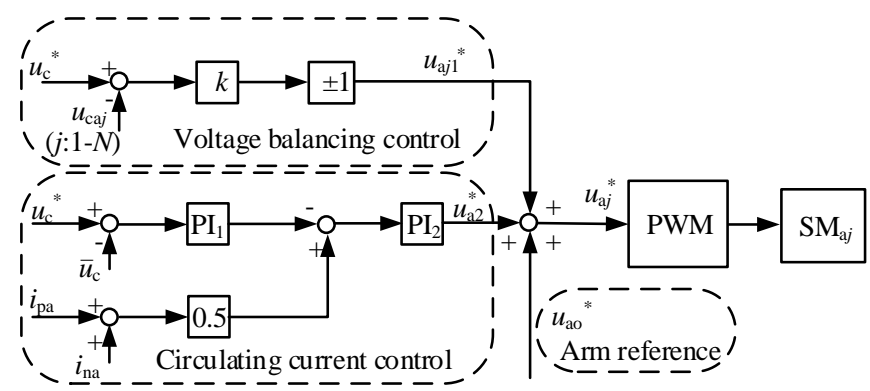

Fig. 4 Voltage balancing and circulating current control diagram of the SM under CPS-PWM

For MV MMCs, the CPS-PWM generates better voltage quality than that of NLM. However, higher hardware requirements for calculation and synchronization are required, due to the extra voltage balancing and circulating current control in each SM, and the high frequency phase-shift modulation among SMs. 
This paper is a post-print of a paper submitted to and accepted for publication in IEEE Transaction on Power Electronics and is subject to Institution of Electrical and Electronic Engineering Copyright. The copy of record is available at IEEE Xplore Digital Library.

\section{PROPOSED NEAREST LEVEL PWM OF MMC}

\section{A. Principle of Proposed NL-PWM}

In order to solve the low-order harmonics problem associated with the NLM and complicated voltage control of CPS-PWM, a new NL-PWM method suitable for MV MMC is proposed in this section.
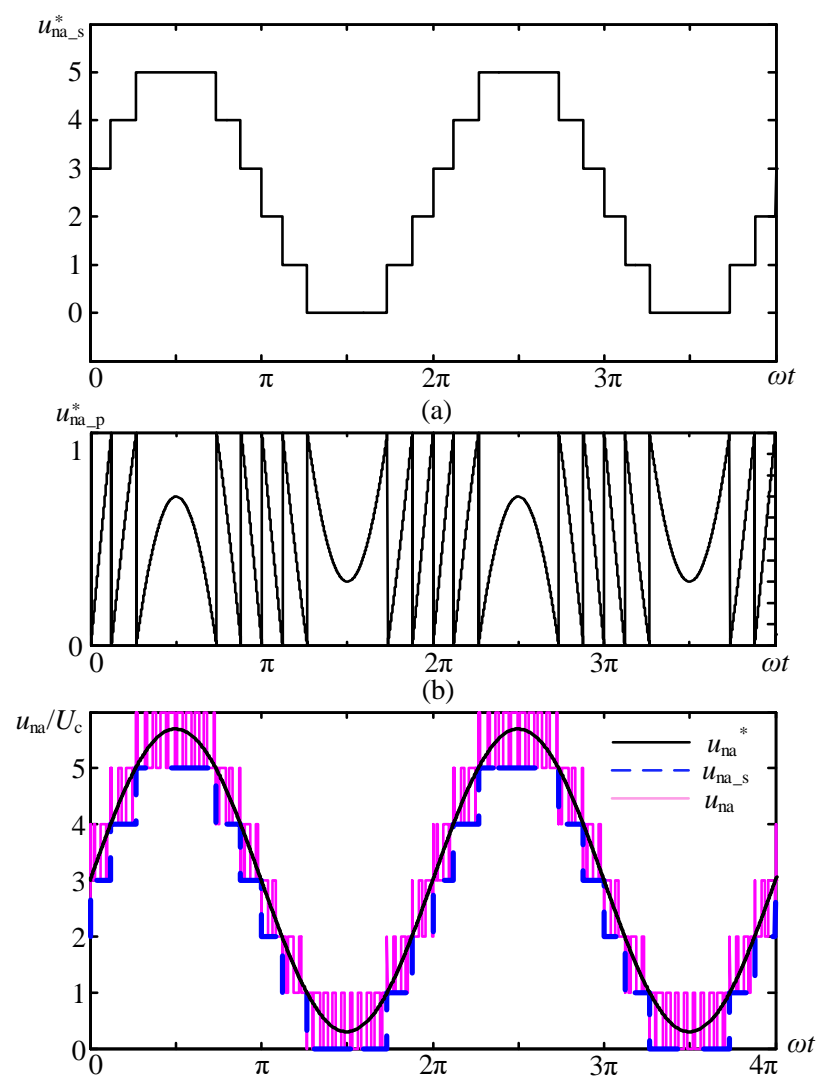

(c)

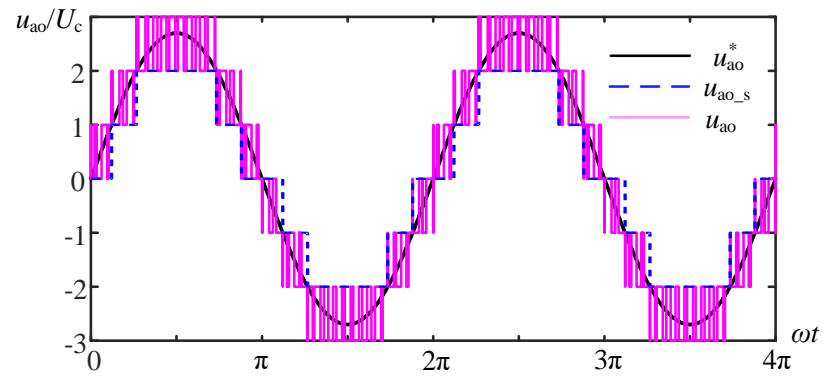

(d)

Fig. 5 Principle of NL-PWM strategy (a) Staircase wave generated by $N_{\text {na }}$ SMs (b) Reference wave of the PWM module (c) Output voltage of lower arm (d) Phase voltage

Fig. 5 illustrates the principle of the proposed NL-PWM for $N=6$. According to (3), the upper and lower arms should each have a PWM module and use opposite-phase references in order to generate the PWM waveform in the phase voltage. The lower arm of phase $a$ is again taken as an example for illustration. A sine wave can be divided into two parts as shown in Fig. 5 (a) and (b). There are $N_{\text {na }}$ SMs operated as inserted mode to generate staircase wave as shown in Fig. 5(a). On this base, only one SM operates 
This paper is a post-print of a paper submitted to and accepted for publication in IEEE Transaction on Power Electronics and is subject to Institution of Electrical and Electronic Engineering Copyright. The copy of record is available at IEEE Xplore Digital Library.

under PWM mode in each arm, and its reference wave is shown in Fig. 5(b). Thus a multi-level PWM waveform is obtained by superposing a two-level PWM wave upon the staircase wave to yield an equivalent sinusoidal output, as shown in Fig. 5 (c). Although the MMC with $N$ SMs per arm can generate $2 N+1$ level while the upper arm using antiphase carriers with that of the lower arm, it leads to larger circulating current due to potential difference in the number of inserted modules of each phase. Therefore, this paper only illustrates $N+1$ level modulation for the MMC. Modulation for the upper arm is almost the same except the reversed phase of the reference wave. According to (3) and the modulation principle mentioned above, the phase voltage is an AC multilevel PWM waveform, as shown in Fig. 5 (d).

For the MV MMC with NL-PWM, the output voltage becomes much closer to the ideal sine wave by introducing a PWM module even with few SMs. The NL-PWM method also inherits the advantage of NLM, i.e., the voltage balancing can be achieved by rotating inserted module sequence according to voltage sorting without extra control in each SM. This not only simplifies the control system but also ensures that the SMs in one arm adopt the same voltage reference as shown in Fig. 5(b). Thus, the noncharacteristic harmonics caused by the different SM voltage references for voltage balancing control under CPS-PWM can be avoided.

\section{B. Implementation of $N L-P W M$}

Under NL-PWM method, each module has three operation modes, i.e. mode 0, 1 and PWM, which are listed in Table II.

TABLE II

\begin{tabular}{cccc}
\multicolumn{4}{c}{ OPERATION MODES OF A SM UNDER NL-PWM } \\
\hline \hline Mode & $\mathrm{S}_{1}$ & $\mathrm{~S}_{2}$ & State \\
\hline 1 & 1 & 0 & inserted \\
0 & 0 & 1 & bypass \\
PWM & $0 \leftrightarrow 1$ & $1 \leftrightarrow 0$ & PWM \\
\hline \hline
\end{tabular}

The number of SMs under mode 1 can be calculated by

$$
\begin{aligned}
& N_{\mathrm{pa}}=\operatorname{floor}\left(\frac{u_{\mathrm{pa}}^{*}}{U_{\mathrm{c}}}\right)=\operatorname{floor}\left(\frac{\frac{1}{2} U_{\mathrm{dc}}-u_{\mathrm{ao}}^{*}}{U_{\mathrm{c}}}\right)=\frac{1}{2} N-\operatorname{floor}\left(\frac{u_{\mathrm{ao}}^{*}}{U_{\mathrm{c}}}\right)-1 \\
& N_{\mathrm{na}}=\operatorname{floor}\left(\frac{u_{\mathrm{na}}^{*}}{U_{\mathrm{c}}}\right)=\operatorname{floor}\left(\frac{u_{\mathrm{ao}}^{*}+\frac{1}{2} U_{\mathrm{dc}}}{U_{\mathrm{c}}}\right)=\frac{1}{2} N+\operatorname{floor}\left(\frac{u_{\mathrm{ao}}^{*}}{U_{\mathrm{c}}}\right)
\end{aligned}
$$

where floor $(\mathrm{x})$ is the function of retuning the largest integer less than or equal to $\mathrm{x} . u_{\mathrm{pa}}{ }^{*}$ and $u_{\mathrm{na}}{ }^{*}$ are the reference wave of the upper arm and lower arm of phase a respectively.

The voltage references of the upper and lower SMs under PWM mode are

$$
\begin{aligned}
& u_{\mathrm{pa} \_ \text {PWM }}^{*}=\left(u_{\mathrm{pa}}^{*}-N_{\mathrm{pa}} U_{\mathrm{c}}\right) / U_{\mathrm{c}}=-\frac{u_{\mathrm{ao}}^{*}}{U_{\mathrm{c}}}+\operatorname{floor}\left(\frac{u_{\mathrm{ao}}^{*}}{U_{\mathrm{c}}}\right)+1 \\
& u_{\mathrm{n} \_ \text {PWM }}^{*}=\left(u_{\mathrm{na}}^{*}-N_{\mathrm{na}} U_{\mathrm{c}}\right) / U_{\mathrm{c}}=\frac{u_{\mathrm{ao}}^{*}}{U_{\mathrm{c}}}-\operatorname{floor}\left(\frac{u_{\mathrm{ao}}^{*}}{U_{\mathrm{c}}}\right)
\end{aligned}
$$

According to (4), (8) and (9), the DC bus voltage can be calculated as 


$$
U_{\mathrm{dc}}=\left(N_{\mathrm{pa}}+u_{\mathrm{pa} \_ \text {PWM }}^{*}+N_{\mathrm{na}}+u_{\mathrm{na} \_ \text {PWM }}^{*}\right) U_{\mathrm{c}}=N U_{\mathrm{c}} .
$$

Therefore, the NL-PWM method can ensure the DC voltage of the MMC to remain at $N U_{\mathrm{c}}$ in any instance while using $N+1$ level modulation. As long as the voltage balancing for each SM is realized, the arm circulating current can be reduced as well. However, for CPS-PWM, extra control loops for voltage balancing and current averaging are required. Similar to NLM, the NL-PWM achieves voltage balancing by sorting the capacitor voltages of each SM in one arm. Therefore, the voltage balancing control of NL-PWM is rather simple and easy to implement.

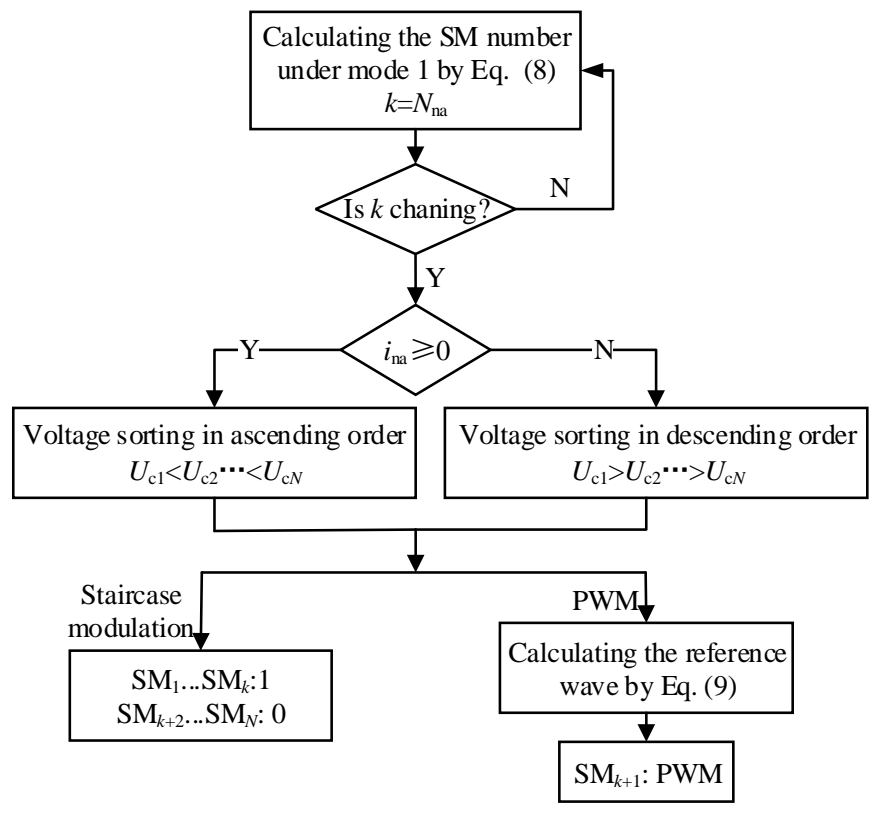

Fig. 6 Modulation diagram of NL-PWM for MMC

Taking the lower arm of phase $a$ as an example, the modulation process of NL-PWM is illustrated in Fig. 6. First, the number of SMs under mode 1, defined as $k$, is calculated using (8). In order to reduce the switching frequency, the voltage sorting and module rotating are activated only when the level of the staircase wave changes instead of during control cycle or PWM cycle. Thus, when the staircase wave level is constant, one of the SMs in an arm is fixed in PWM mode, and its mode will be changed while next voltage sorting occurs. As the SM operating in PWM mode switches between modes 1 and 0 , it has less capacitor charging or discharging current than other SMs operating in mode 1. Therefore, the $k$ SMs with the lowest/highest voltages should operate in mode 1 for increased changing/discharging, whereas the $(k+1)$ th SM is selected as PWM module. For example, while $i_{\text {na }} \geq 0$, the SMs operate in charging state, so the $k$ SMs with lower capacitor voltages are selected to operate in mode 1 . while the $(k+1)$ th SM is identified as the PWM module, and the rest of SMs operate in mode 0.

Due to the voltage sorting and module rotating when the staircase level changes in NLM, and the PWM module in NL-PWM has less charging/discharging current, the voltage ripple and required capacitor size of NL-PWM will similar to those of NLM with the same number of SMs. However, the switching loss of NL-PWM is slightly higher than that of NLM as one SM is operated 
This paper is a post-print of a paper submitted to and accepted for publication in IEEE Transaction on Power Electronics and is subject to Institution of Electrical and Electronic Engineering Copyright. The copy of record is available at IEEE Xplore Digital Library.

in PWM mode for reducing the current distortion. However, its total switching loss is still much lower than that of a two-level PWM converter..

\section{Harmonic analysis of the MMC with NL-PWM}

The capacitor voltages of the SMs are assumed to be balanced and constant in the following harmonic analysis. According to the principle of NL-PWM, the output multilevel PWM waveform of the MMC can be regarded as the superposition of a $N$ - 1 level staircase wave and a two-level PWM wave, i.e.

$$
u_{\mathrm{na}}=u_{\mathrm{stair}}+u_{\mathrm{PWM}} \text {. }
$$

Therefore, the harmonic analysis of $u_{\mathrm{na}}$ is carried out with $u_{\text {stair }}$ and $u_{\mathrm{PWM}}$ separately.

According to the double Fourier transform for carrier based PWM method [19]-[20], the PWM wave can be expressed as

$$
\begin{gathered}
u(t)=u(x, y)=\frac{A_{00}}{2}+\sum_{n=1}^{\infty}\left\{A_{0 n} \cos (n y)+B_{0 n} \sin (n y)\right\}+\sum_{m=1}^{\infty}\left\{A_{m 0} \cos (m x)+B_{m 0} \sin (m x)\right\}+\sum_{m=1}^{\infty} \sum_{n= \pm 1}^{ \pm \infty}\left[A_{m n} \cos (m x+n y)+B_{m n} \sin (m x+n y)\right] \\
A_{m n}+j B_{m n}=C_{m n}=\frac{1}{2 \pi^{2}} \int_{-\pi}^{\pi} \int_{-\pi}^{\pi} u(x, y) e^{j(m x+n y)} d x d y
\end{gathered}
$$

where $y=\omega_{\mathrm{r}} t$ and $x=\omega_{\mathrm{c}} t, \omega_{\mathrm{r}}$ and $\omega_{\mathrm{c}}$ are the angular frequencies of the reference wave and carrier wave, respectively.

To obtain analytical expressions for the harmonics, the valid integral interval for $x$ and $y$ should be determined first, and then the amplitude of harmonics can be calculated by (13).

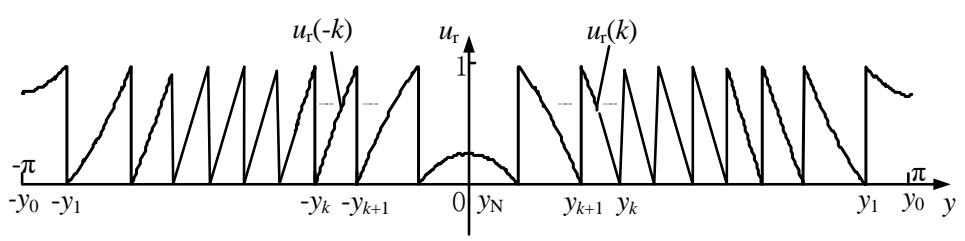

Fig. 7 Reference wave of the PWM module in the lower arm

The reference wave of the PWM module in the lower arm, namely $u_{\mathrm{r}}$, is shown in Fig. 7, which can be divided into $2 N$ segments. The switching instants are $\pm y_{0}, \ldots \pm y_{k} \ldots \pm y_{N}$, where $y_{0}=\pi, y_{N}=0$, and segment number $k$ is the number of SMs in mode 1 .

The per-unit reference wave of segment $\pm k$ can be expressed as

$$
u_{\mathrm{r}}(k)=u_{\mathrm{r}}(-k)=M \cos y+\frac{N}{2}-k \quad y \in\left[-y_{k},-y_{k+1}\right] \cup\left[y_{k+1}, y_{k}\right] \quad k=0,1 \mathrm{~L} \quad N-1
$$

where $M=U_{\text {ao_m }} / U_{\mathrm{c}}$. $U_{\mathrm{ao} \_\mathrm{m}}$ is the peak value of $u_{\mathrm{ao}}^{*}$ and the capacitor voltage $U_{\mathrm{c}}$ is the voltage base.

The switching instant $y_{\mathrm{k}}$ can be calculated by

$$
y_{k}=\arccos \left(\frac{k+1-N / 2}{M}\right) .
$$




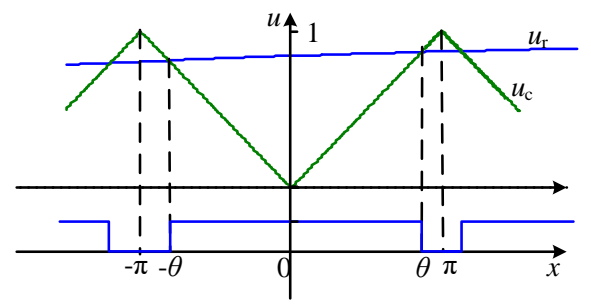

Fig. 8 PWM modulation process in one carrier cycle

The PWM modulation process in one carrier cycle is shown in Fig. 8, where $-\theta, \theta$ are the switching on/off points of the PWM module in one cycle, and the PWM wave in one cycle can be expressed as

where

$$
u_{\mathrm{PWM}}(x)= \begin{cases}1 & x \in\left[\begin{array}{ll}
-\theta & \theta
\end{array}\right] \\
0 & x \in\left[\begin{array}{ll}
-\pi & -\theta
\end{array}\right) \cup\left(\begin{array}{ll}
\theta & \pi
\end{array}\right]\end{cases}
$$

$$
\theta=\pi\left[M \cos y+\frac{N}{2}-k\right] \quad y \in\left[-y_{k},-y_{k+1}\right] \cup\left[y_{k+1}, y_{k}\right]
$$

According to (12)-(17), $u_{\mathrm{PWM}}$ can be derived as

$$
u_{\mathrm{PWM}}=\frac{A_{00}}{2}+\sum_{n=1}^{\infty} A_{0 n} \cos n y+\sum_{m=1,3,5 \mathrm{~L}}^{\infty} A_{m 0} \cos (m x)+\sum_{m=2,4,6 \mathrm{~L}}^{\infty} \sum_{n= \pm 1, \pm 3, \pm 5 \mathrm{~L}}^{ \pm \infty} A_{m n_{-} 1} \cos (m x+n y)+\sum_{m=1,3,5 \mathrm{~L}}^{\infty} \sum_{n= \pm 2, \pm 4, \pm 6 \mathrm{~L}}^{ \pm \infty} A_{m n_{-} 2} \cos (m x+n y)
$$

where

$$
\begin{gathered}
A_{00}=N-\frac{2}{\pi} \sum_{k=0}^{N-1} y_{k} \\
A_{0 n}=\left\{\begin{array}{l}
M-\frac{2}{\pi} \sum_{k=0}^{N-1} \sin y_{k} \quad n=1 \\
-\frac{2}{n \pi} \sum_{k=0}^{N-1} \sin n y_{k} \quad n \neq 1
\end{array}\right. \\
A_{m 0}=\frac{8}{m \pi^{2}} \sum_{l=1,3,5 \mathrm{~L}}^{\infty} \sin \left(\frac{l \pi}{2}\right) J_{l}(m \pi M) \times \frac{1}{l}\left[\sin \left(\frac{l \pi}{2}\right)+2 \sum_{k=N / 2}^{<M>+N / 2-2}(-1)^{k-\frac{N}{2}+1} \sin \left(l y_{k}\right)\right] \\
A_{m n-2}=\frac{4}{m \pi^{2}} \sum_{l=1,3,5 \mathrm{~L}}^{\infty} \sin \left(\frac{l \pi}{2}\right) J_{l}(m \pi M) \times\left\{\frac { 1 } { n + l } \left[\sin (n+l) \frac{\pi}{2}+2 \sum_{k=N / 2}^{<M>+N / 2-2}(-1){ }^{k-\frac{N}{2}+1} \sin \left(\frac{n \pi}{2}\right) J_{n}(m \pi M)\right.\right. \\
+\frac{1}{n-l}\left[\sin (n-l) \frac{\pi}{2}+2 \sum_{k=N / 2}^{<M>+N / 2-2}(-1) y_{k}\right]
\end{gathered}
$$

where $\left\langle M>\right.$ represents the smallest integer greater than $M, J_{n}(\mathrm{x})$ is the $n$-order Bessel function.

The Fourier series of the staircase wave in the lower arm is derived as

$$
u_{\text {stair }}=\frac{1}{\pi} \sum_{k=0}^{N-1} y_{k}+\sum_{n=1}^{\infty}\left(\frac{2}{n \pi} \sum_{k=0}^{N-1} \sin n y_{k}\right) \cos n y
$$


Substituting (18) and (24) to (11), the output voltage of the lower arm is expressed as

$$
u_{\mathrm{na}}=\frac{N}{2}+M \cos \omega_{\mathrm{r}} t+\sum_{m=1,3,5 \mathrm{~L}}^{\infty} A_{m 0} \cos \left(m \omega_{\mathrm{c}} t\right)+\sum_{m=2,4,6 \mathrm{~L}}^{\infty} \sum_{n= \pm 1, \pm 3, \pm 5 \mathrm{~L}}^{ \pm \infty} A_{m n_{-} 1} \cos \left(m \omega_{\mathrm{c}} t+n \omega_{\mathrm{s}} t\right)+\sum_{m=1,3,5 \mathrm{~L}}^{\infty} \sum_{n= \pm 2, \pm 4, \pm 6 \mathrm{~L}}^{ \pm \infty} A_{m n_{-} 2} \cos \left(m \omega_{\mathrm{c}} t+n \omega_{\mathrm{s}} t\right)
$$

For the $N+1$ level modulation, the phase voltage has the same frequency spectrum with the lower arm voltage waveform except the DC component, which is expressed as

$$
u_{\mathrm{ao}}=M \cos \omega_{\mathrm{r}} t+\sum_{m=1,3,5 \mathrm{~L}}^{\infty} A_{m 0} \cos \left(m \omega_{\mathrm{c}} t\right)+\sum_{m=2,4,6 \mathrm{~L}}^{\infty} \sum_{n= \pm 1, \pm 3, \pm \mathrm{L}}^{ \pm \infty} A_{m n_{-} 1} \cos \left(m \omega_{\mathrm{c}} t+n \omega_{\mathrm{r}} t\right)+\sum_{m=1,3,5 \mathrm{~L}}^{\infty} \sum_{n= \pm 2, \pm 4, \pm 6 \mathrm{~L}}^{ \pm \infty} A_{m n_{-} 2} \cos \left(m \omega_{\mathrm{c}} t+n \omega_{\mathrm{r}} t\right) .
$$

Likewise, the output voltage of phase $b$ can be derived as

$$
\begin{aligned}
& u_{\mathrm{bo}}=M \cos \left(\omega_{\mathrm{r}} t-\frac{2}{3} \pi\right)+\sum_{m=1,3,5 \mathrm{~L}}^{\infty} A_{m 0} \cos \left(m \omega_{\mathrm{c}} t\right)+\sum_{m=2,4,6 \mathrm{~L}}^{\infty} \sum_{n= \pm 1, \pm 3, \pm 5 \mathrm{~L}}^{ \pm \infty} A_{m n_{-} 1} \cos \left(m \omega_{\mathrm{c}} t+n \omega_{\mathrm{r}} t-n \frac{2}{3} \pi\right) . \\
& +\sum_{m=1,3,5 \mathrm{~L}}^{\infty} \sum_{n= \pm 2, \pm 4, \pm 6 \mathrm{~L}}^{ \pm \infty} A_{m n_{-} 2} \cos \left(m \omega_{\mathrm{c}} t+n \omega_{\mathrm{r}} t-n \frac{2}{3} \pi\right)
\end{aligned}
$$

Then the Fourier series of the line voltage $u_{\mathrm{ab}}$ is given as

$$
\begin{aligned}
& u_{\mathrm{ab}}=u_{\mathrm{ao}}-u_{\mathrm{bo}}=-\sqrt{3} M \sin \left(\omega_{\mathrm{r}} t-\frac{\pi}{3}\right)-2 \sum_{m=2,4,6 \mathrm{~L}}^{\infty} \sum_{n= \pm 1, \pm 3, \pm 5 \mathrm{~L}}^{ \pm \infty} A_{m n_{-} 1} \sin \left(m \omega_{\mathrm{c}} t+n \omega_{\mathrm{r}} t-\frac{n \pi}{3}\right) \sin \frac{n \pi}{3} \\
& -2 \sum_{m=1,3,5 \mathrm{~L}}^{\infty} \sum_{n= \pm 2, \pm 4, \pm 6 \mathrm{~L}}^{ \pm \infty} A_{m n_{-} 2} \sin \left(m \omega_{\mathrm{c}} t+n \omega_{\mathrm{r}} t-\frac{n \pi}{3}\right) \sin \frac{n \pi}{3}
\end{aligned}
$$

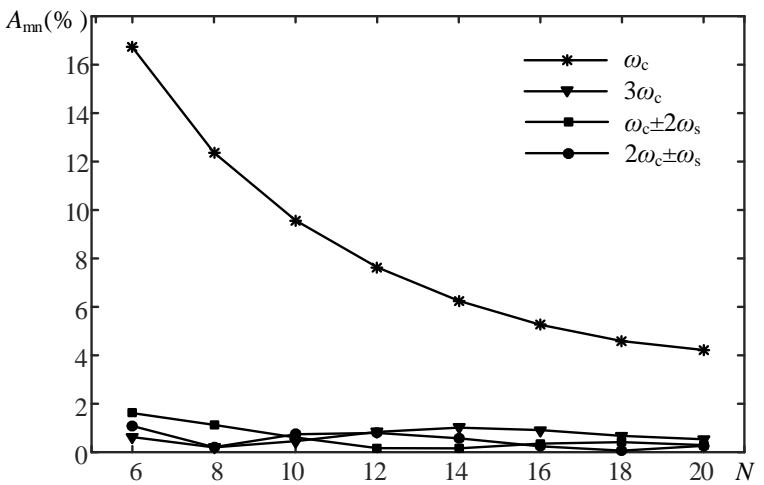

Fig. 9 The main harmonic components of the phase voltage by NL-PWM with different $N$

The main harmonic components of the phase voltage with different $N$ while $f_{\mathrm{c}}=2000 \mathrm{~Hz}$ and $f_{\mathrm{r}}=50 \mathrm{~Hz}$ are calculated according to above harmonic analytical expressions and shown in Fig. 9. The harmonic characteristics of the NL-PWM are summarized as follows.

(1) There is no low-order harmonics. The phase voltage contains odd carrier frequency harmonics $\left(\right.$ e.g. $\left.\omega_{\mathrm{c}}, 3 \omega_{\mathrm{c}} \cdots\right)$, sideband harmonics near carrier frequency (e.g. $\left.\omega_{\mathrm{c}} \pm 2 \omega_{\mathrm{r}}, \omega_{\mathrm{c}} \pm 4 \omega_{\mathrm{r}} \cdots, 2 \omega_{\mathrm{c}} \pm \omega_{\mathrm{r}}, 2 \omega_{\mathrm{c}} \pm 3 \omega_{\mathrm{r}} \cdots\right)$. The line voltage does not contain the harmonics of $m \omega_{\mathrm{c}}(m=1,2,3 \cdots)$.

(2) As seen in Fig. 9, the harmonic of $\omega_{\mathrm{c}}$ is the most prominent harmonic component in the phase voltage spectrum, and the other harmonics are all less than $2 \%$ even with $N=6$. As the line voltage has no $\omega_{\mathrm{c}}$ harmonic, its total harmonic distortions $(T H D)$ 
This paper is a post-print of a paper submitted to and accepted for publication in IEEE Transaction on Power Electronics and is subject to Institution of Electrical and Electronic Engineering Copyright. The copy of record is available at IEEE Xplore Digital Library.

stays small even for the MMC with very few modules. However, for CPS-PWM, it has larger sideband harmonics caused by the multi-carrier modulation which also appear in line voltages.

(3) The specific harmonic content and THD of the MMC with different $N$ and $\omega_{\mathrm{c}}$ can be easily calculated using a program based on above harmonic analytical expressions instead of complicated MMC simulation, thus providing a theoretical basis for MV MMC design.

\section{SimUlATION STUDIES}

\section{A. Comparisons of different modulation methods}

To demonstrate the benefits of the proposed NL-PWM method for the MMC with fewer modules, comparisons of the output voltage and current are conducted with other two methods, i.e., NLM and CPS-PWM. The simulation parameters are listed in Table III.

TABLE III

PARAMETERS OF THE MMC SYSTEM FOR SIMULATION

\begin{tabular}{ll}
\hline \hline \multicolumn{1}{c}{ Items } & \multicolumn{1}{c}{ Parameters } \\
\hline DC bus rated voltage & $U_{\mathrm{dc} \_\mathrm{N}}=6000 \mathrm{~V}$ \\
Rated Power & $P_{\mathrm{N}}=150 \mathrm{~kW}$ \\
Number of SMs in one arm & $N=6$ \\
Arm inductance & $L_{\mathrm{s}}=10 \mathrm{mH}$ \\
Capacitor of SM & $C=3000 \mu \mathrm{F}$ \\
SM capacitor rated voltage & $U_{\mathrm{CN}}=1000 \mathrm{~V}$ \\
AC load & $R_{\mathrm{L}}=100 \Omega$ \\
Reference wave Frequency & $L_{\mathrm{L}}=20 \mathrm{mH}$ \\
Carrier frequency of NL-PWM & $f_{\mathrm{r}}=50 \mathrm{~Hz}$ \\
Carrier frequency of CPS-PWM & $f_{\mathrm{cl}}=2000 \mathrm{~Hz}$ \\
Modulation ratio & $f_{\mathrm{c} 2}=333 \mathrm{~Hz}$ \\
\hline
\end{tabular}

The waveforms of the line voltage $u_{\mathrm{ab}}$ and its corresponding spectrum, the phase current $i_{\mathrm{a}}$, circulating current $i_{\mathrm{za}}$ and capacitor voltage $U_{\mathrm{c}}$ under the modulations of NL-PWM, NLM and CPS-PWM are compared in Figs. 10 (a), (b) and (c) respectively, whereas the THDs of the line voltages and phase currents are listed in Table IV. As can be seen from the line voltage spectrums, there are notable low-order voltage harmonics under NLM. NL-PWM and CPS-PWM both have high-order harmonics, however, NL-PWM has smaller harmonics around $\omega_{\mathrm{c}}$. The THDs of the voltage and current under NL-PWM are the smallest of the three modulations due to no low-order harmonics and smaller high-order harmonics. The amplitudes of the circulating current and capacitor voltage variation are nearly the same under these three modulations. As a result, the output current under NL-PWM is smoother than those under NLM and CPS-NLM.

TABLE IV

\begin{tabular}{cccc}
\multicolumn{4}{c}{ THDS OF OUTPUT VOLTAGE AND CURRENT $(\%)$} \\
\hline \hline \multicolumn{1}{c}{ Items } & NL-PWM & NLM & CPS-PWM \\
\hline Line voltage & 9.2 & 12 & 14.18 \\
Phase current & 2.64 & 9.30 & 4.69 \\
\hline \hline
\end{tabular}



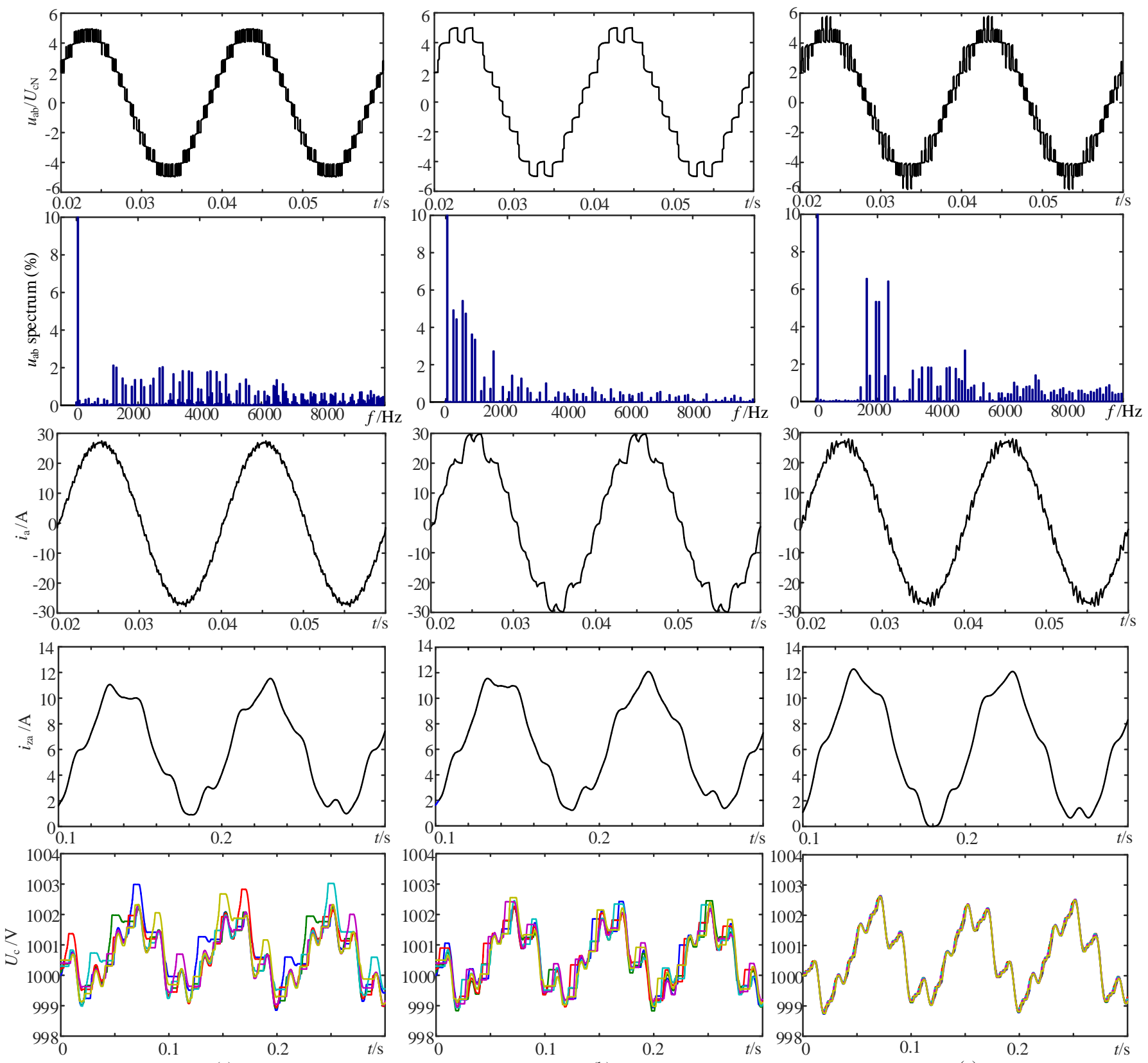

(a)

(b)

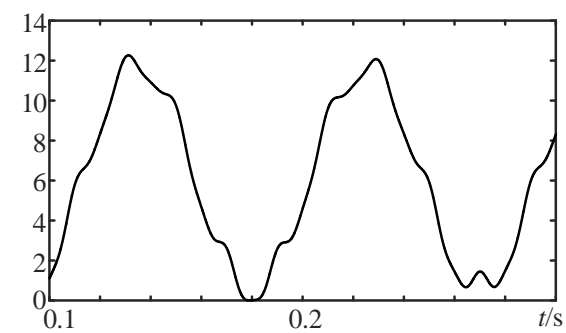

Fig. 10 Simulated comparisons of the MMC output voltage and current with different modulations (a) NL-PWM (b) NLM (c) CPS-PWM

\section{B. Verification of the harmonic analytical expression}

To verify the correctness of the presented harmonic expressions under NL-PWM, the calculated and simulated harmonic contents of the MMC phase voltage with different $N$ are compared in Table $\mathrm{V}$, and their spectra of $N=6$ are shown in Fig. 11. Unlike the theoretical calculation, the capacitor voltages are not constant and contain variations in the simulation with practical parameters. However, as the capacitor voltages are well balanced, the effect of their small variations to the harmonic spectrum is negligible. It can be seen that, the theoretical calculations correspond the simulation results well for different $N$. For example, when $N=6$, the $T H D$ of the phase voltage is $21.3 \%$ by simulation and $21.18 \%$ by theoretical calculation, whereas the largest harmonics 
This paper is a post-print of a paper submitted to and accepted for publication in IEEE Transaction on Power Electronics and is subject to Institution of Electrical and Electronic Engineering Copyright. The copy of record is available at IEEE Xplore Digital Library.

of $\omega_{\mathrm{c}}$ are $16.76 \%$ and $16.72 \%$ by simulation and theoretical calculation, respectively. It can also be seen that, the THD of the phase voltage is inversely proportional to $N$, e.g., the $T H D$ of $N=6$ is almost 2 times of the $T H D$ of $N=12$.
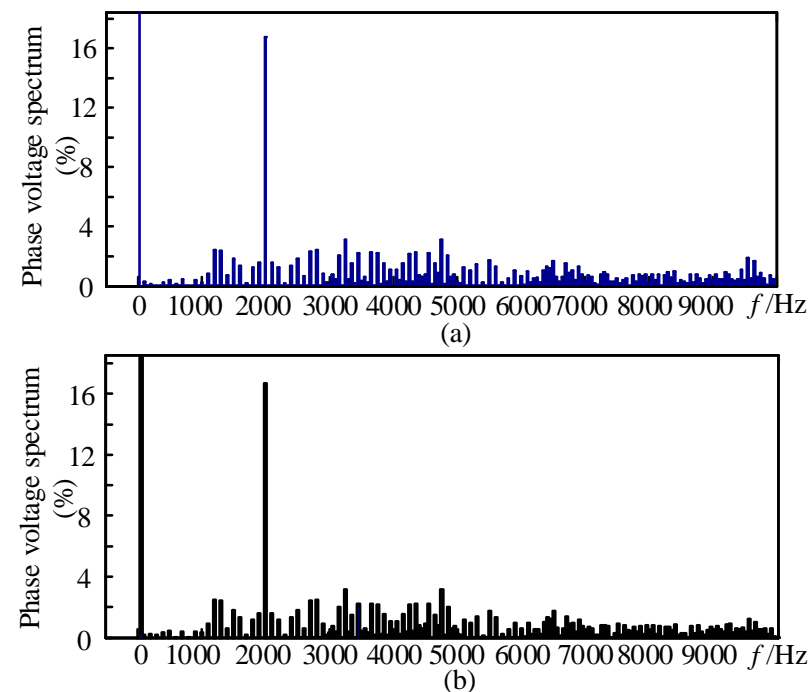

Fig. 11 Comparison of simulated and calculated spectrums of the phase voltage under NL-PWM (a) Simulated spectrum (b) Calculated spectrum

TABLE V

\begin{tabular}{l|c|c|c|c|c|c|c|c}
\multicolumn{9}{|c|}{ HARMONIC RATIOS OF THE PHASE VOLTAGE OF NL-PWM WITH DIFFERENT $N$} \\
\hline \hline $\begin{array}{l}\text { Harmonic } \\
\text { frequency }\end{array}$ & $\begin{array}{c}\text { Simulation } \\
(\%)\end{array}$ & $\begin{array}{c}\text { Calculation } \\
(\%)\end{array}$ & $\begin{array}{c}\text { Simulation } \\
(\%)\end{array}$ & $\begin{array}{c}\text { Calculation } \\
(\%)\end{array}$ & $\begin{array}{c}\text { Simulation } \\
(\%)\end{array}$ & $\begin{array}{c}\text { Calculation } \\
(\%)\end{array}$ & $\begin{array}{c}\text { Simulation } \\
(\%)\end{array}$ & $\begin{array}{c}\text { Calculation } \\
(\%)\end{array}$ \\
\hline$\omega_{\mathrm{c}}$ & 16.76 & 16.72 & 12.16 & 12.37 & 7.48 & 7.63 & 6.48 & 6.25 \\
$\omega_{\mathrm{c}} \pm 2 \omega_{\mathrm{s}}$ & 1.55 & 1.61 & 1.16 & 1.13 & 0.22 & 0.17 & 0.28 & 0.16 \\
$2 \omega_{\mathrm{c}} \pm \omega_{\mathrm{s}}$ & 1.08 & 1.08 & 0.20 & 0.21 & 0.75 & 0.80 & 0.38 & 0.57 \\
$T H D$ & 21.3 & 21.18 & 16.51 & 16.06 & 10.66 & 10.34 & 9.22 & 8.89 \\
\hline \hline
\end{tabular}

V. EXPERIMENTAL VERIFICATION

To further verify the feasibility of the proposed NL-PWM method in a practical system, an MMC experimental platform is set up. The structure of the experimental platform is shown in Fig. 12, and the main parameters of the system are listed in Table VI. The DC side of the MMC is supplied by a diode rectifier, and the AC side is an inductive load. Thus the MMC operates as an inverter to transmit power from the DC side to the AC load.

Fig. 13 shows the experimental waveforms of the SM voltages in one arm and the phase voltage. There is only one SM operates at PWM mode at any instant, and the mode switching and voltage sorting occur at the instant of level changing. Fig. 14 shows the experimental results of the capacitor voltages of the SMs in one arm. Although only one SM operates at PWM mode in one arm, the voltage balancing is realized by SM rotation according to voltage sorting. Figs. 15 (a) and (b) show the experimental results of the three-phase voltages and currents with the modulation ratio changing from 0.6 to 0.9 , under NLM and NL-PWM respectively. The obvious current distortion $\left(T H D_{\mathrm{i}}=6.43\right)$ can be seen with the 7-level staircase voltages under NLM, whereas a much lower current distortion $\left(T H D_{\mathrm{i}}=1.37\right)$ is achieved when fed by the 7-level PWM voltages under NL-PWM, which are consistent with the theoretical analysis and simulation results. 


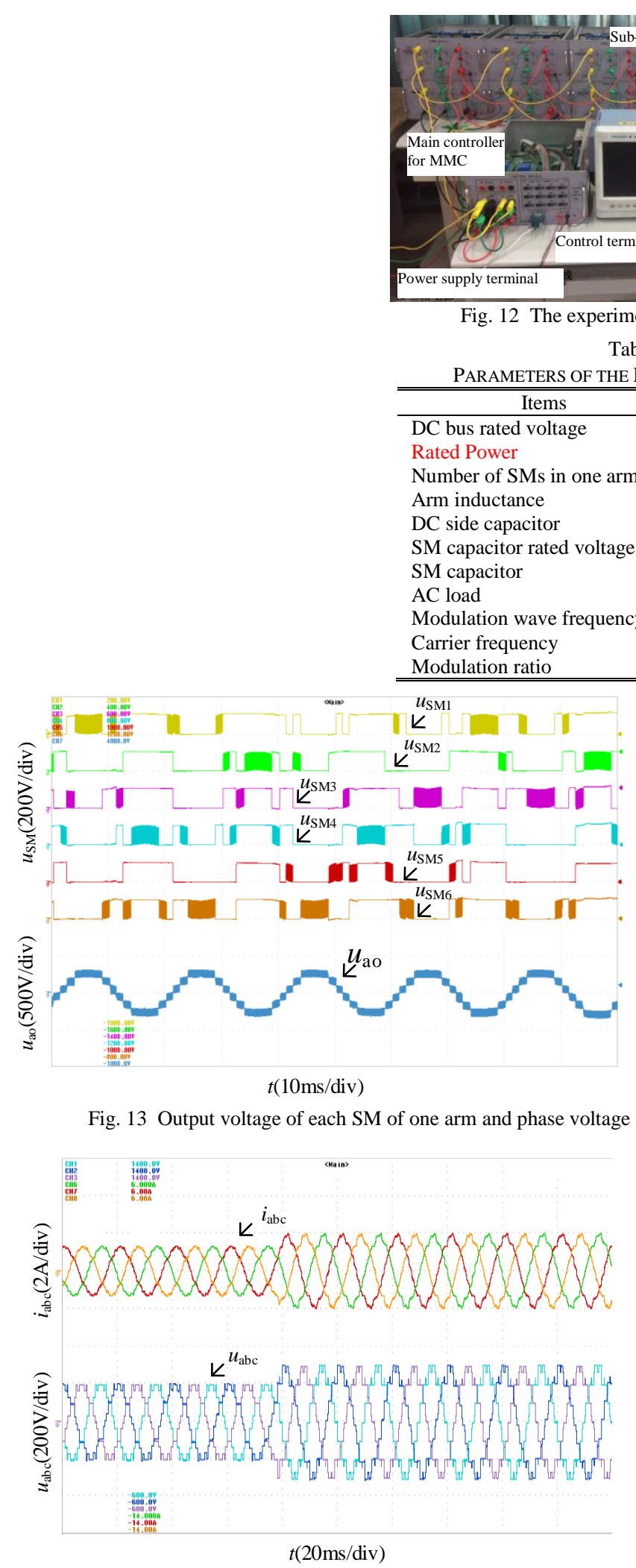

(a)

Table VI

\begin{tabular}{ll}
\multicolumn{2}{c}{ PARAMETERS OF THE EXPERIMENTAL SYSTEM } \\
\hline \hline \multicolumn{1}{c}{ Items } & \multicolumn{1}{c}{ Parameters } \\
\hline DC bus rated voltage & $U_{\mathrm{dc}}=600 \mathrm{~V}$ \\
Rated Power & $P_{\mathrm{N}}=5 \mathrm{~kW}$ \\
Number of SMs in one arm & $N=6$ \\
Arm inductance & $L_{\mathrm{s}}=1.7 \mathrm{mH}$ \\
DC side capacitor & $C_{\mathrm{dc}}=28.4 \mathrm{mF}$ \\
SM capacitor rated voltage & $U_{\mathrm{cN}}=100 \mathrm{~V}$ \\
SM capacitor & $\mathrm{C}=2.84 \mathrm{mF}$ \\
AC load & $L_{\mathrm{L}}=80 \mathrm{mH} R_{\mathrm{L}}=150 \Omega$ \\
Modulation wave frequency & $f_{\mathrm{r}}=50 \mathrm{~Hz}$ \\
Carrier frequency & $f_{\mathrm{c}}=2000 \mathrm{~Hz}$ \\
Modulation ratio & $M=0.9$ \\
\hline \hline
\end{tabular}

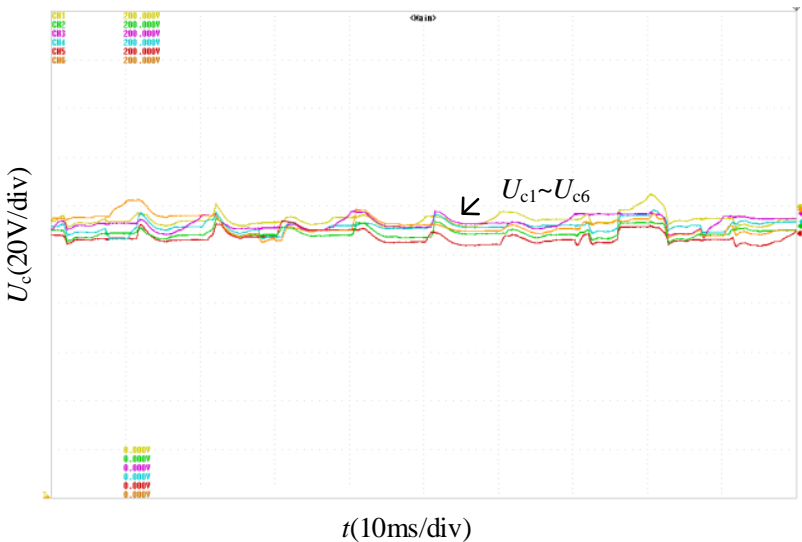

Fig. 14 Capacitor voltages of each SM in one arm

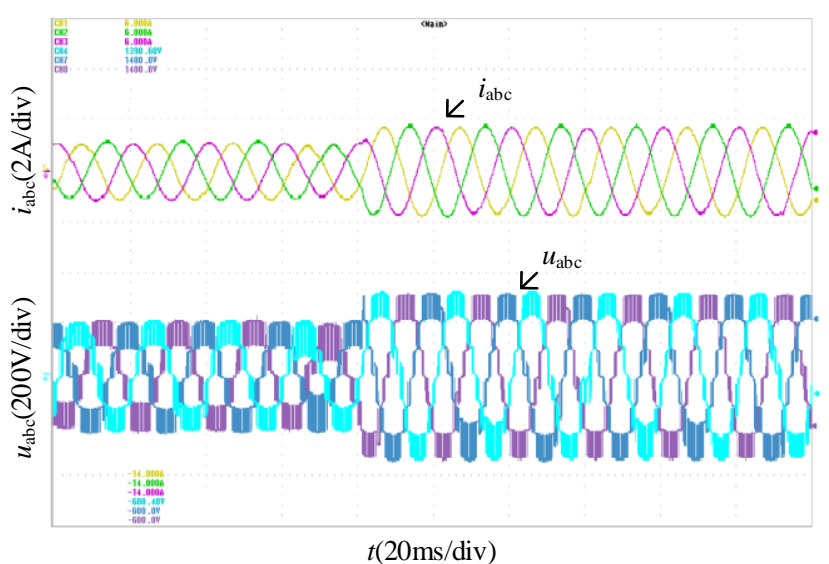

(b)

Fig. 15 Waveforms of three-phase currents and voltages of the MMC (a) NLM (b) NL-PWM 
This paper is a post-print of a paper submitted to and accepted for publication in IEEE Transaction on Power Electronics and is subject to Institution of Electrical and Electronic Engineering Copyright. The copy of record is available at IEEE Xplore Digital Library.

\section{CONCLUSIONS}

This paper proposes a NL-PWM method for the MMC with fewer SMs applied to the DC distribution grids, and its validity and feasibility are tested by simulation and experimental studies. In contrast to traditional modulations, the NL-PWM has the advantages of simple voltage balancing control and better harmonic characteristics over NLM and CPS-PWM. Therefore, it is suited for MMC used in MVDC distribution networks. The harmonic expressions of the MMC output phase and line voltages under NL-PWM are obtained by means of double Fourier transform, which shows good harmonic characteristics of the proposed modulation method. The analytic expressions can provide a theoretical basis for practical applications of NL-PWM on MV MMC while choosing the switching frequency and module number to meet the distribution grid requirements.

\section{REFERENCES}

[1] L. G. Franquelo, J. Rodriguez, J. I. Leon, S. Kouro, R. Portillo and M. A. M. Prats, "The age of multilevel converters arrives," IEEE Ind. Electron. Mag. , vol. 2, no. 2, pp. 28-39, Jun. 2008.

[2] S. Debnath, J. Qin, B. Bahrani, M. Saeedifard and P. Barbosa, "Operation, Control, and Applications of the Modular Multilevel Converter: A Review," IEEE Trans.Power Electron., vol. 30, no. 1, pp. 37-53, Jan. 2015.

[3] A. M. Trzynadlowski and S. Legowski, "Minimum-loss vector PWM strategy for three-phase inverters," IEEE Trans.Power Electron., vol. 9, no. 1, pp. 26-34, Jan. 1994.

[4] A. M. Hava, R. J. Kerkman and T. A. Lipo, "A high-performance generalized discontinuous PWM algorithm," IEEE Trans. Ind. Appl., vol. 34, no. 5, pp. 10591071, Sept./Oct. 1998.

[5] M. Guan, Z. Xu and Hairong Chen, "Control and modulation strategies for modular multilevel converter based HVDC system," in IECON-IES, Melbourne, VIC, 2011, pp. 849-854.

[6] P. Hu and D. Jiang, "A Level-Increased Nearest Level Modulation Method for Modular Multilevel Converters," IEEE Trans.Power Electron. , vol. 30, no. 4, pp. 1836-1842, Apr. 2015.

[7] Y. Deng, M. Saeedifard and R. G. Harley, "An improved nearest-level modulation method for the modular multilevel converter," in APEC, Charlotte, NC, 2015, pp. 1595-1600.

[8] L. Lin, Y. Lin, Z. He, Y. Chen, J. Hu and W. Li, "Improved Nearest-Level Modulation for a Modular Multilevel Converter With a Lower Submodule Number," IEEE Trans.Power Electron., vol. 31, no. 8, pp. 5369-5377, Aug. 2016.

[9] Y. Zhou, D. Jiang, P. Hu, J. Guo, Y. Liang and Z. Lin, "A Prototype of Modular Multilevel Converters," IEEE Trans.Power Electron., vol. 29, no. 7, pp. 32673278, Jul. 2014.

[10] M. Hagiwara and H. Akagi, "Control and Experiment of Pulsewidth-Modulated Modular Multilevel Converters," IEEE Trans.Power Electron. , vol. 24, no. 7, pp. 1737-1746, Jul. 2009.

[11] M. Hagiwara and H. Akagi, "PWM control and experiment of modular multilevel converters," in PESC, Rhodes, GRC, 2008 , pp. 154-161.

[12] X. Li, Q. Song, J. Li and W. Liu, "Capacitor voltage balancing control based on CPS-PWM of Modular Multilevel Converter," in ECCE, Phoenix, AZ, 2011, pp. $4029-4034$. 
This paper is a post-print of a paper submitted to and accepted for publication in IEEE Transaction on Power Electronics and is subject to Institution of Electrical and Electronic Engineering Copyright. The copy of record is available at IEEE Xplore Digital Library.

[13] F. Deng and Z. Chen, "A Control Method for Voltage Balancing in Modular Multilevel Converters," IEEE Trans.Power Electron., vol. 29, no. 1, pp. 66-76, Jan. 2014.

[14] Q. Tu, Z. Xu and L. Xu, "Reduced Switching-Frequency Modulation and Circulating Current Suppression for Modular Multilevel Converters," IEEE Trans. Power Del., vol. 26, no. 3, pp. 2009-2017, Jul. 2011.

[15] M. Zhang, L. Huang, W. Yao and Z. Lu, "Circulating Harmonic Current Elimination of a CPS-PWM-Based Modular Multilevel Converter With a Plug-In Repetitive Controller," IEEE Trans.Power Electron., vol. 29, no. 4, pp. 2083-2097, Apr. 2014.

[16] S. Lu, L. Yuan, K. Li and Z. Zhao, "An Improved Phase-Shifted Carrier Modulation Scheme for a Hybrid Modular Multilevel Converter," IEEE Trans.Power Electron., vol. 32, no. 1, pp. 81-97, Jan. 2017.

[17] K. Li, L. Yuan, Z. Zhao, S. Lu and Y. Zhang, "Fault-Tolerant Control of MMC With Hot Reserved Submodules Based on Carrier Phase Shift Modulation," IEEE Trans.Power Electron., vol. 32, no. 9, pp. 6778-6791, Sept. 2017.

[18] B. Tai, C. Gao, X. Liu and Z. Chen, "A Novel Flexible Capacitor Voltage Control Strategy for Variable-Speed Drives With Modular Multilevel Converters,"IEEE Trans.Power Electron., vol. 32, no. 1, pp. 128-141, Jan. 2017.

[19] J. Liu, Y. Sun, Y. Li and C. Fu, "Theoretical harmonic analysis of cascaded H-bridge inverter under hybrid pulse width multilevel modulation," IET Power Electronics, vol. 9, no. 14, pp. 2714-2722, Nov. 2016.

[20] Y. Li, Y. Wang and B. Q. Li, "Generalized Theory of Phase-Shifted Carrier PWM for Cascaded H-Bridge Converters and Modular Multilevel Converters," IEEE J. Emerg. Sel. Topics Power Electron., vol. 4, no. 2, pp. 589-605, Jun. 2016. 